\title{
Evaluation of the Effect of Citrus Lemon L. "Enter" on Blood Pressure in Hypertensive Patients
}

\author{
Zeynep Güneş Özünal ${ }^{1}$, Iliriana Alloqi Tahirbegolli ${ }^{2}$ and Ali Yağız Üresin ${ }^{2}$ \\ 1 Medical Pharmacology Department, Faculty of Medicine, Maltepe University, İstanbul 34845, Turkey \\ 2 Medical Pharmacology Department, Faculty of Medicine, Istanbul University, İstanbul 34093, Turkey
}

\begin{abstract}
This study aimed to evaluate the effectiveness of lemon juice to lower blood pressure. Twenty nine patients were included. Blood pressure measurements were done before (basal) and 5th, 15th and at 30th minute after lemon juice or equal volume of water intake. When compared with the basal values, patients who were given lemon juice had lower systolic blood pressure at 5 th, 15 th and 30 th min. Control patients who were given water had lower systolic blood pressure at 5 th and 15 th, and diastolic blood pressure at 15 th min. When compared with the basal values, patients who were given lemon juice had lower heart rate at 15 th and 30th and control patients who were given water had decreased heart rate at 5th, 15th and 30th min. Both lemon juice and water decreased systolic blood pressure within first 15 th minutes possibly due to doctor and environmental silent effect. These results do not support lemon juice intake.
\end{abstract}

Key words: Hypertension, citrus lemon, blood pressure.

\section{Introduction}

High BP (blood pressure) is one of the most important cardiovascular risk factors worldwide. Lifestyle modifications such as physical activity and healthy diet are recommended beside pharmacological treatment. Complementary medicine use is very common among hypertensive patients. A systematic review of 26 surveys in 13 countries showed that the mean prevalence rate of complementary and alternative medicine (CAM) use was $31.4 \%$ [1]. Also the prevalence of CAM use in Turkey varied greatly among individual chronic diseases, being most prevalent in hypertension (63.8\%) [2]. In Turkey $72.5 \%$ of hypertensive patients are using alternative therapy, $40 \%$ being lemon juice [3]. Preclinical study with SHR (spontaneous hypertensive rats) investigated the effect of lemon juice and concluded oral intake of lemon juice resulted with blood pressure decrease and proposed flavonoids with inhibitory effect on ACE (angiotensin converting enzyme) activity may be

Corresponding author: Zeynep Güneş Özünal, M.D., asst. prof., research fields: clinical pharmacology, hypertension. responsible for the effect [4]. Nevertheless evidence is lacking on acute effects of lemon juice in hypertensive patients. In this study we aimed to investigate Citrus Lemon L. "Enter" effect on blood pressure, heart rate, and drug adherence. We also questioned possible adverse effects of lemon juice intake.

\section{Method and Materials}

Patients who admitted to Istanbul University Istanbul Faculty of Medicine Pharmacology Research Polyclinic with at least 6 months of hypertension history and treated with at least one antihypertensive medication, 29 subjects were enrolled to study. Exclusion criteria were diabetes mellitus, gastrointestinal hemorrhages history or related drug use, infections, dementia, terminal illness, allergy, recent significant medical diagnosis, breast feeding or pregnancy, patients that are hungry, systolic blood pressure $(\mathrm{SBP}) \geq 160 \mathrm{mmHg}$, diastolic blood pressure (DBP) $\geq 100 \mathrm{mmHg}$.

The study design was single-blinded randomized placebo-controlled parallel single dose study. The study was approved by the Human Research Ethics 
Committee at the Istanbul University Istanbul Faculty of Medicine. Consenting eligible patients were randomly allocated to lemon juice or water groups using a computer-generated random number table. Patients' eligibility was assessed at their visit with the research nurse at their usual practice. Patient who enrolled in trial is entered into a quiet room, not frequented by other people. The room was dominated with white color, which resembles hospital room, the patient sits in a comfortable chair, and is asked to turn off the phone. After 15 minutes the researcher enters in the room and starts the measurements and treatment according to protocol. Sociodemographic data and drugs that are used questioned. Weight and height is measured. Repeating blood pressure measurements and heart rate per minute were performed. The researcher doesn't stay all the time in the room and talks to patients only about the procedure of research measurements. To standardize lemon juice content, the same Citrus Lemon L. "Enter" tree and same juice volume is used, kept and transferred in appropriate conditions. Preparation and drinking dishes were made of glass. Lemon juice is prepared $50 \mathrm{~mL}$ hand-squeezed lemon and $150 \mathrm{~mL}$ water, total of 200 $\mathrm{ml}$ at $25 \%$ concentration fresh just before drinking. No sugar is added. Primary outcome measures were SBP and DBP. Blood pressure and heart rate are measured using OMRON 705 IT. All measures are checked strictly according to study protocol. BP in the beginning of investigation is checked from both arms and then the readings were continued with the arm where the BP were higher. Sitting measurements are done before and 5th, 15th and at 30th minute after treatment. Visual analog score is used to assess the stressfulness. The day after treatment the patient is questioned by phone call and asked if there were any adverse effects and is checked if antihypertensive medications were taken.

\section{Results and Discussion}

Age and BMI index was not statistically significant between lemon juice group and water group (Table 1). Lemon juice decreased sitting systolic blood pressure at 5th, 15th and 30th minutes and decreased heart rate at 15th and 30th when compared to preintervention values. Water decreased systolic blood pressure at 5th and 15th and decreased diastolic at 15th minutes and decreased heart rate at 5th, 15th and 30th minutes when compared to preintervention values (Table 2).

Visual analog score was 56 and 72 in lemon juice

Table 1 Patient gender, age and BMI (body mass index) in lemon juice and water groups.

\begin{tabular}{|c|c|c|}
\hline & Lemon juice $(\mathrm{n}=17)$ & Water $(n=12)$ \\
\hline \multicolumn{3}{|l|}{ Gender } \\
\hline Male (\%) & $10(58.8)$ & $9(75.0)$ \\
\hline Female (\%) & $7(41.2)$ & $3(25.0)$ \\
\hline Age (Mean \pm SD) & $54.82 \pm 9.35$ & $52.75 \pm 9.69$ \\
\hline BMI (Mean \pm SD) & $29.22 \pm 4.75$ & $29.86 \pm 3.46$ \\
\hline
\end{tabular}

Table 2 Systolic, diastolic BP (blood pressure) and pulse, before and 5, 15 and 30 minutes after lemon juice or water.

\begin{tabular}{|c|c|c|c|c|c|c|c|}
\hline & $\begin{array}{l}\text { Before treatment } \\
\text { Mean } \pm \text { SD }\end{array}$ & $\begin{array}{l}5 \text { minutes after } \\
\text { treatment Mean } \pm \\
\text { SD }\end{array}$ & $p$ & $\begin{array}{l}15 \text { minutes after } \\
\text { treatment Mean } \pm \\
\text { SD }\end{array}$ & $p$ & $\begin{array}{l}30 \text { minutes after } \\
\text { treatment Mean } \pm \\
\text { SD }\end{array}$ & $p$ \\
\hline $\begin{array}{l}\text { Lemon juice } \\
\text { Systolic BP }\end{array}$ & $135.19 \pm 15.11$ & $129.19 \pm 14.34$ & 0.022 & $129.33 \pm 12.67$ & 0.014 & $127.96 \pm 10.92$ & 0.006 \\
\hline Diastolic BP & $78.46 \pm 8.74$ & $77.35 \pm 8.9$ & 0.355 & $77.6 \pm 7.97$ & 0.453 & $77.96 \pm 6.44$ & 0.679 \\
\hline Pulse & $72.62 \pm 10.0$ & $71.02 \pm 7.85$ & 0.136 & $70.0 \pm 7.37$ & 0.021 & $69.82 \pm 7.88$ & 0.011 \\
\hline $\begin{array}{l}\text { Water } \\
\text { Systolic BP }\end{array}$ & $128.46 \pm 16.03$ & $124.44 \pm 17.21$ & 0.041 & $120.58 \pm 15.46$ & 0.002 & $123.03 \pm 15.75$ & 0.084 \\
\hline Diastolic BP & $76.99 \pm 10.82$ & $74.03 \pm 12.64$ & 0.91 & $72.25 \pm 9.93$ & 0.023 & $73.47 \pm 11.55$ & 0.084 \\
\hline Pulse & $68.69 \pm 6.0$ & $67.16 \pm 5.71$ & 0.028 & $65.58 \pm 5.21$ & 0.013 & $65.37 \pm 6.05$ & 0.021 \\
\hline
\end{tabular}


and water groups respectively. The day after treatment phone interview resulted with any adverse effect is found. All patients $(n=29)$ took their antihypertensive medications.

Both lemon juice and water decreased systolic blood pressure within first 15 th minutes possibly because of sitting effect. At 30th minute lemon juice effect, decrease in systolic blood pressure $8 \mathrm{mmHg}$ differentiated from water. Any decrease in diastolic blood pressure is shown in lemon juice group. Lemon juice effects on blood pressure can be due to taste, odour or components in juice. Similar with our results, 30 days with intravenous $500 \mathrm{mg} /$ day ascorbic acid treatment also resulted with systolic blood pressure decrease [5]. In our study the possible effect can be caused by ascorbic acid component expected to be 20-23 mg, quite lower than the mentioned trial [6]. Ascorbic acid is reported to be one of the dietary supplements related with blood pressure decrease possibly preventing nitric oxide breakdown [7]. Citrus interdonato juice also includes flavonoids [8]. Citrus lemon includes various natural bioactive compounds not only ascorbic acid but also minerals, dietary fiber, essential oils, organic acids, carotenoids, and flavonoids [9].

\section{Conclusions}

In hypertension therapy, life style modifications are readily recommended to improve blood pressure control including fruit consumption. Drugs are already shown to be effective and safe. An alternative therapy in hypertension should have evidence on not only decrease in blood pressure but also on end organ damage failure. Safety issues such as possible drug interactions, adverse affects are important. Natural does not mean safe to recommend patients. Although lemon juice is frequently used among Turkish hypertensive population, our study results are not supporting its use. The blood pressure decrease would be expected different in hypertensive patients who have higher blood pressure levels but in this study we did not recruit these less controlled hypertensive patients because of ethical considerations. Adverse effects and drug adherence is not differed between the groups but we did not include more risky population like acid related disease diagnose or diabetes mellitus. The patients are told in consent that they are going to be phoned next day about drug intake and this might increased their drug adherence. Our results on adverse effects and drug adherence may not represent the real time settings. The patients who frequently use lemon juice to decrease blood pressure should be questioned about drug adherence and blood pressure control.

\section{Acknowledgement}

We thank to Alata Horticultural Research Institute, Erdemli, Mersin, Turkey for providing Citrus lemon L. "Enter" and MD Bernard Tahirbegolli for the statistical consultancy. We thank to Mr David F. Chapman (Language Editor, Istanbul Faculty of Medicine) for editing the manuscript.

\section{References}

[1] Lorenc, A., Ilan-Clarke, Y., Robinson, N., and Blair, M. 2009. "How Parents Choose to Use CAM: A Systematic Review of Theoretical Models." BMC Complement Altern Med. 9: 9.

[2] Mollaoğlu, M., and Aciyurt, A. 2013. "Use of Complementary and Alternative Medicine among Patients with Chronic Diseases." Acta Clin Croat. 52: 181-8.

[3] Adibelli, Z., Dilek, M., and Akopolat, T. 2009. "Lemon Juice as an Alternative Therapy in Hypertension in Turkey." Int J Cardiol. 135 (2): 58-9.

[4] Miyake, Y., Kuzuya, K., Ueno, C., Katayama, N., Hayakawa, T., Tsuge, H., and Osawa, T. 1998. "Suppressive Effect of Components in Spontaneously Hypertensive Rats in Lemon Juice on Blood Pressure." Food Sci. Technol. Int. Tokyo 4 (1): 29-32.

[5] Duffy, S. J., Gokce, N., Holbrook, M., Huang, A., Frei, B., Keaney, J. F., and Vita, J. F. 1999. "Treatment of Hypertension with Ascorbic Acid." Lancet 354: 2048-9.

[6] Ballentine, R. 1941. "Determination of Ascorbic Acid in Citrus Fruit Juices.” Ind Engen. Chem Anal. 13: 89.

[7] Wilburn, A. J., King, D. S., Glisson, J., Rockhold, R. W., and Wofford, M. R. 2004. "The Natural Treatment of Hypertension.” The Journal of Clinical Hypertension 6 (5): 
242-8.

[8] Caristi, C., Bellocco, E., Panzera, V., Toscano, G., Vadalà, R., and Leuzzi, U. 2003. "Flavonoids Detection by HPLC-DAD-MS-MS in Lemon Juices from Sicilian Cultivars." J. Agric. Food Chem. 51: 3528-34.
[9] González-Molina, E., Domínguez-Perles, R., Moreno, D. A., and García-Viguera, C. 2010. "Natural Bioactive Compounds of Citrus Limon for Food and Health." Journal of Pharmaceutical and Biomedical Analysis 51: $327-45$. 